\title{
A Public Transport System Based Sensor Network for Road Surface Condition Monitoring
}

\author{
Kasun De Zoysa \\ kasun@cmb.ac.lk \\ Gihan P. Seneviratne \\ gps@ucsc.cmb.ac.lk
}

\author{
Chamath Keppitiyagama \\ chamath@ucsc.cmb.ac.lk \\ W. W. A. T. Shihan \\ shihanuoc@yahoo.com
}

University of Colombo School of Computing

35, Reid Avenue, Colombo - 07, Sri Lanka

\begin{abstract}
A well maintained road network is a must for the economic development and the well being of people in any country. Unfortunately, most developing countries do not poses such road networks. While the lack of funds is mainly to blame for not building new road networks and maintaining the existing ones the lack of proper monitoring and reporting system is a major contributory factor for the dilapidated condition of road networks in third world countries. A case in point is the road network in Sri Lanka; Sri Lanka has an extensive road network that spans the country and new roads are being built every day, yet even the roads in the capital city are not maintained properly. The lack of a monitoring and reporting mechanism is apparent in this case.

We propose a public transport system based sensor network to monitor road surface condition. We are currently building such a network called BusNet to monitor environmental pollution and that system can be extended for road surface condition monitoring by adding acceleration sensor boards to the system.
\end{abstract}

\section{Categories and Subject Descriptors}

C.2.1 [Computer-Communication Networks]: Network Architecture and Design; J.2 [Physical Sciences and Engineering]: Physics

\section{General Terms}

Design, Experimentation

\section{Keywords}

Sensor Networks, Ad Hoc Networks, Delay Tolerant Networks

\section{INTRODUCTION}

A good, well maintained road network helps economic development of a country as well as the well being of its citizens. Vehicles consume less fuel on a well maintained road surface. Smooth movement of traffic on such a surface also cuts the traveling time

Permission to make digital or hard copies of all or part of this work for personal or classroom use is granted without fee provided that copies are not made or distributed for profit or commercial advantage and that copies bear this notice and the full citation on the first page. To copy otherwise, to republish, to post on servers or to redistribute to lists, requires prior specific permission and/or a fee.

NSDR'07, August 27, 2007, Kyoto, Japan.

Copyright 2007 ACM 978-1-59593-787-2/07/0008 ...\$5.00.

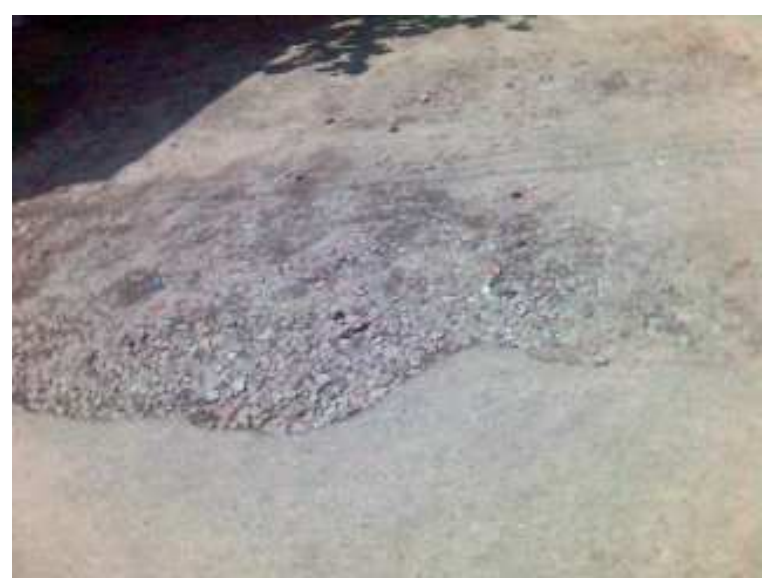

Figure 1: A deteriorated road surface.

and helps to reduce the otherwise wasted person hours. A good road network also helps to reduce the traffic congestion and this in turn helps to reduce the environmental pollution.

Unfortunately, most developing countries do not have well maintained road networks there by hindering the development of those countries. The lack of funds is certainly a factor that contributes to the deteriorated road networks, but the lack of proper monitoring and reporting systems is also a major contributor.

In Sri Lanka there is an extensive road network spanning the island. New roads are being built and the existing roads are being upgraded every day. However, we observe that even the roads in the capital city are in deteriorated condition (Figure 1). A tropical climate with two heavy monsoon seasons and the movement of heavy vehicles contribute to the dilapidated condition of the road surfaces. The lack of monitoring and reporting system delays the resurfacing and maintaining even when the funds are available. Availability of continuous monitoring system can help in two ways.

1. Triggering the maintenance system in to action before the road condition deteriorates beyond the acceptable level. A timely warning mechanism can reduce the cost of repairing. The maintainers can also take preventive measures and control the deterioration until proper repairing can be done.

2. Providing data to researchers to study how the road surface condition deteriorates. Such a study enables better planning of future networks. 
Continuous monitoring of surface condition of a road network that spans thousands of kilometers is a complex resource intensive task. It is impractical to deploy a large number of sensors along the road network and it is also prohibitively expensive to use a purpose built vehicle mounted sensor system deployed just for this purpose. However, we observe that in Sri Lanka, as well as in many other countries, there is a public transport system that uses the road network extensively. The public transport buses are in a unique position to carry sensors to monitor the road surface condition. We propose to build a sensor network based on public transport network to monitor road surface condition. The public transport buses can easily carry commodity off the shelf wireless sensors that can be used for road surface condition monitoring. We are already developing a sensor network called BusNet [11] which uses wireless sensors mounted on public transport buses to monitor environmental pollution. The BusNet is extended to include sensors to monitor road surface condition.

In this paper we describe the proposed system to monitor the the road surface condition. First, we describe the BusNet and then we describe how we propose to extend the BusNet with acceleration sensors to monitor the surface condition.

\section{BUSNET}

BusNet is a sensor network initially designed to monitor environmental pollution using sensors mounted on public transport buses. In designing the BusNet for this initial application we realized that the same system can be used for road surface condition monitoring with just minor modifications and in fact that it is an ideal network for that purpose.

The design of the BusNet is based on the observation that environmental monitoring systems need a large number of sensor nodes spread over a large area to be effective. Citing a study by Arora et al. [1], Tanenbaum et al. [9] note that the range of the thermal, humidity, infrared, and magnetic sensors are less than their radio ranges. The implication of this is that these systems require a large number of sensor nodes to collect data and to maintain the connectivity in order to transfer the data to collection centers. The SensorScope [2] project uses 110 sensors to cover an area of $1200 \times 600 \mathrm{~m}$.

A sensor system that has a large number of sensors spread over a large area poses several problems.

- It would be quite expensive to build a system with a large number of sensors when each sensor is expensive. While sensor boards and motes are relatively inexpensive they are still quite expensive for a developing country.

- It is not easy to maintain and manage a system consisting of a large number of sensors. The regular replacement of batteries and replacement of faulty sensors are costly and labor intensive tasks. In addition, locating faulty sensors to replace them is not an easy task.

- Protecting a large number of sensors scattered throughout a large terrain is a daunting task. Sensors can be damaged by animals, heavy rains, and there is also a possibility of vandalizing.

We observe that most countries have a public transport system that spans the country. This is the case in Sri Lanka. Since most people cannot afford private vehicles there is a high demand for the public transport system. The bus and the train networks have central points (such as regional bus/train stations) from which regional transport networks span out. There are bus and train routes

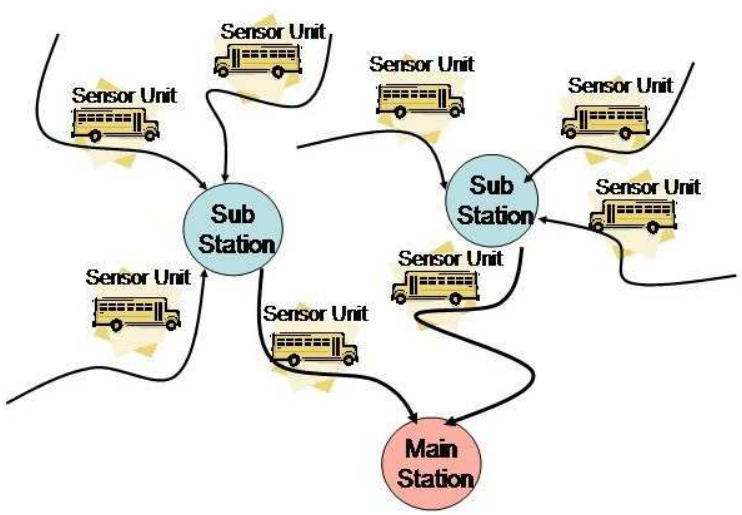

Figure 2: BusNet System Architecture

inter-connecting these regional stations as well as central hubs in major cities. This forms a large public transport network. In BusNet, we use several sensors mounted on the vehicles of the public transport system as a replacement for a network consisting of large number of sensors. These vehicle mounted moving sensors gather data that covers a large geographical area. When the buses arrive at bus stations, which also function as data collection centers, gathered data are transferred over a wireless link to the collection point. Data gathered in regional collection points are transferred to buses traveling between the regional centers and the main collection center. In this scenario the public transport system functions as a data delivery network as well as the data collection network.

We assume that the gathered data are not required in real time and this assumptions holds for the environment monitoring application as well as for the road surface monitoring application. Data collected for both these applications are used to study long term changes and the warnings triggered by the two systems do not require reactions from the interested/responsible parties within minutes or hours-both these applications are delay tolerant.

\subsection{BusNet Architecture}

Figure 2 depicts the architecture of the BusNet. The BusNet has three main components; Sensor Units, Sub Stations, and a Main Station.

The sensor unit consists of a Crossbow MICAz mote [3], and several sensor boards including a GPS sensor board. The sensor boards other than the GPS sensor board depend on the type of data to be gathered (temperature, carbon monoxide level etc.). This sensor unit will be mounted on top of a bus and it gets power from the battery of the bus. Other than getting power from the bus's battery we do not expect any cooperation from the bus driver or the bus operators. The sensors gather environmental data along the bus route. The collected data will be stored together with the GPS coordinates of the collection points. In the current prototype these data points will be stored in the memory of the sensor mote. However, we expect to connect a storage devise, such as a flash drive, to the sensor unit in future.

The sub stations are the collection nodes located at the regional bus stations. Regional bus routes span out from the sub stations. Buses going out and coming towards the sub stations collect the temperature and carbon monoxide levels at regular intervals and store them together with the GPS coordinates. Once they reach the sub station they transmit the data over a wireless network to the sub station. The sub stations route these collected data to the main station over the bus network. It uses buses going to other regional 
bus stations to send the data to the main station, and before reaching the main station the collected data may have to go through several sub stations, which act as routers.

Since we do not expect the cooperation of the buses other than carrying the sensor units on them, we cannot assume that buses will wait in the station until the completion of the data transfer. To handle this we are developing a protocol which prioritize the data transfer in application specific manner. In the environmental monitoring application the prioritization algorithm ensures that a larger area is covered. In this case, the sensor unit initially transfers the data collected at $1 \mathrm{Km}$ intervals and next transfers data collected at $500 \mathrm{~m}$ interval and so on. It prioritizes the data based on the spatial coordinates so that the data collected over a large area is transferred first and only covers the points in between if the time permits. In addition to this, when there is more than one bus transferring data, the station must make sure that it collects data from all the buses in a round robin fashion to ensure that it collects data from all the routes fairly.

The proposed data transfer protocol does not stop to retransmit lost packets; that is, if a packet is lost its retransmission is delayed and retransmission starts only after the first attempt transmission of all the packets. Therefore, retransmission takes place only if the bus stays long enough to retransmit. Priority is given to the first attempt packets to ensure transmission of data to cover a large terrain as much as possible.

The main station is similar to the sub stations but it has a direct connection to the data processing center and it does not have to route data to other stations.

\subsection{Advantages}

The BusNet has several advantages over a traditional sensor network system and it solves the problems associated with large a number of sensors spread over a large terrain as follows.

- The BusNet uses few moving sensors as a replacement for a large number of sensors. This brings down the cost of the monitoring system.

- Since the sensors eventually come to a Sub Station or the Main Station, maintenance work such as battery replacement and the replacement/repairing of faulty sensors can be done at these stations.

- Buses are parked in secured areas when they are not in operation and this reduces the possible theft and damages to the sensors.

\subsection{Limitations}

BusNet also has several limitations. The data collected from few moving sensors obviously have a lesser degree of accuracy than the data collected using a large number of stationary sensors. We conjecture that the data collected from moving sensors can be extrapolated to obtain results that are closer to the ones obtained through stationary sensors. The monitoring system is restricted to an area adjacent to the roads serviced by the bus routes, but the environmental pollution is highest by the roads and cities and those are the areas that requires continuous monitoring. The limited coverage area of the BusNet is not a problem for the road surface monitoring system because the very roads that we want to monitor are being serviced by the bus network.

\section{ROAD SURFACE MONITORING}

The above described BusNet was originally designed to collect environmental data. However, in designing the BusNet we realized that it can easily function as a sensor network to monitor the road surface condition.

To monitor road surface condition the sensor motes are mounted with accelerations sensors in addition to the sensors that gather environmental data. The acceleration sensor boards are capable of measuring both the vertical and the horizontal acceleration. We conjecture that the normal component of the acceleration has a correlation with the road surface condition. An obvious example is that a bus going over a pot hole on the road. In this case there would be a significant change in the normal component of the acceleration.

In addition to the vertical acceleration the horizontal acceleration in the opposite direction to the movement of the vehicle, which indicates a decrease in the speed, also can be taken as an indication of worsening road condition in certain cases. For example, the bus has to reduce speed over a rough section of the road.

This is a highly simplified view of the road condition monitoring system. The acceleration readings are affected by other factors. We describe them in Section 4.

The collected acceleration readings are transmitted over the BusNet to the central collection point at the Main Station. Data travel in vehicles in the BusNet and therefore it is a high latency network. Because of that the collection center does not get the data in real time. However, road condition deteriorates over a long period. Therefore, a few hours delay in sending out alerts is acceptable. In other words, this is a delay tolerant application and the the BusNet is an ideal network for it.

In the initial design, the buses transfer collected data in increasing level of distance granularity. The rationale for this is to transfer data to cover as much area as possible before the bus leaves a Sub or the Main Station. However, this transfer protocol has to be changed for the road surface monitoring application to transfer data points that indicate high level of road deterioration with high priority.

\section{ESTIMATING THE SURFACE CONDITION}

The task of the BusNet is to simply gather the data and transfer them to the collection point at the Main Station. The sensor units do not process data. However, the usefulness of the BusNet for road surface condition monitoring depends on the usefulness of the gathered data (acceleration) in estimating the surface condition. To address this issue we are currently developing an analytical model to estimate road surface condition based on the acceleration data. This model is developed with the help of the Department of Physics, University of Colombo.

As mentioned before the road surface monitoring system is based on our hypothesis that there is a direct correlations between the acceleration and the condition of the road surface. However, the acceleration readings are also affected by certain attributes of the vehicle that the sensor system is mounted on.

The suspension system of the vehicle has an impact on the acceleration readings. A good suspension system can dampen the impact of the road surface condition on the vehicle. The weight distribution of the vehicle also has a bearing on the acceleration. In addition the size of the tires also affect the impact of the road condition on the vehicle. For example, a vehicle with large tires may not even feel the impact of a pot hole on the road.

For any given vehicle it may be possible to factor out above mentioned attributes. We expect that this can be generalized for a given vehicle type, but obviously there would be a reduction in the accuracy of the interpolated results.

The sudden changes in the speed of the vehicle is also an indicator of the rough road surface condition. Drivers tend to slow 
down when they judge that the patch of road ahead to be a rough one. However, how soon they can take the decision to slow down depends on the vision of the driver as well as the experience. Therefore, the actual weight of the horizontal component of the acceleration on the roughness indicator depends on the skill of the driver.

We do not expect the system to give an exact measure of the condition of the road, but we expect that it would be able to indicate several discrete levels of deterioration. Our initial aim is to derive a reasonable estimator of road surface condition based on the acceleration values which can be easily measured by the sensor unit.

The impact of the road surface condition on the vehicle (and hence on its speed and the acceleration) depends on the complex interaction of several parameters. For example the impact of the visibility and the vision of the driver on the change of the speed and the direction of the vehicle is far too complex to be captured by an analytical model. With the understanding gained by developing the analytical model we intend to explore the possibility of using a neural network to estimate the roughness of the road surface based on the acceleration.

As mentioned before the sensor system does not process the data. The motes are not designed to perform heavy calculations. The sensor system simply reports the acceleration readings to the processing center over the BusNet. The data are processed at the processing center. However, the sensor units on the BusNet must be given the threshold value of acceleration that indicates significant deterioration of the road surface so that the data collection can be triggered by that value. Since the sensor unit is constrained by the memory available such a triggering point is needed to conserve the storage space.

\section{CHALLENGES}

The sensor should be able to withstand the tropical conditions of Sri Lanka-they are to be mounted on top of the buses. However, ruggedizing the sensor units is beyond the scope of this research and we will not consider this for the prototype we are designing. A real world deployment certainly needs a robust sensor unit.

We will have to face logistical problems in deploying the BusNet because we need certain amount of support from the authorities who manage the public transport system. In addition the data gathered would be useless unless the authorities responsible for maintaining the road system establishes a system to monitor the collected data and respond accordingly.

There are several limitations of the road surface monitoring system. The above description assumes that the vehicles drive over the deteriorated patches in the road. While it is not always possible, vehicles tend to avoid the rough surfaces and we miss the opportunity to detect the deteriorated surface. However, in such a situation there will be significant change in the horizontal component of the acceleration. While such a change in the horizontal components of the acceleration does not necessarily indicate a rough patch of road-it may indicate a traffic jam - a large number of such readings over time for a particular point in the road indeed indicates the possibility of deteriorated surface condition. Vehicles may not always be able to avoid rough patches and because of that it is also possible to get direct data about that location once in a while giving more confidence to the horizontal acceleration data.

As we mentioned before the impact on the vehicles traveling on rough surfaces is dampened by the suspension system of the vehicle. Even though it is possible to calibrate the sensor system for a particular vehicle, the suspension system also changes over time. Ideally, the system should be able to calibrate itself dynamically to account for such changes. One possibility is to do this calibration while the vehicle is on a patch of road of known surface condition.

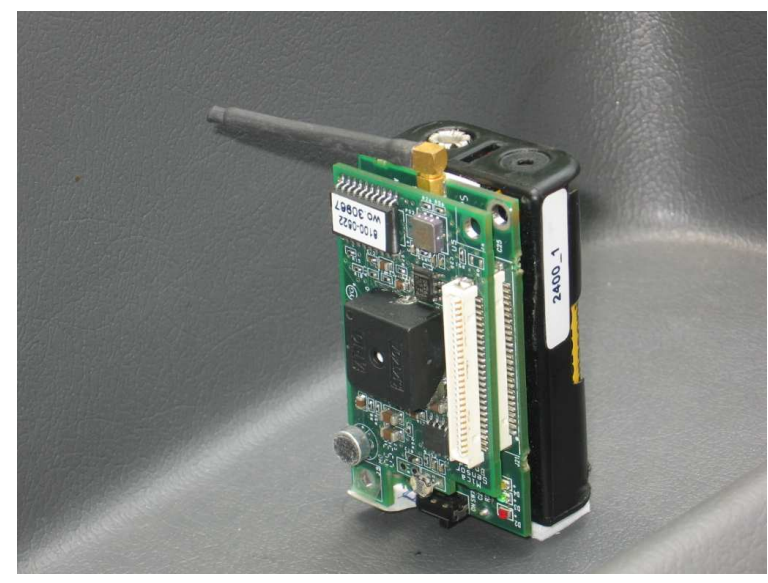

Figure 3: A MICAz mote with a sensor board mounted inside a car.

However, this would be a complex task and we assume for the time being that the change of the suspension system and other conditions of the vehicle occur over a long time period.

To interpolate the surface condition from the acceleration data we also have to take in to account the weight of the vehicle that the sensor system is mounted on. Since we are using the public transport system the weight of the buses vary widely depending on he number of passengers that they carry. Since we cannot measure the change of the weight of the vehicle due to this the interpolated results may vary in accuracy.

The vertical acceleration can also change because of the condition of the terrain on which the road is built. For example, a mountain road. However, we can expect a good road on such a mountain area to give gradually changing acceleration readings (very low frequency) and deteriorated road patches to indicate sudden sharp changes in speed.

These challenges and limitations open up several interesting avenues of research on moving sensors.

\section{CURRENT STATUS}

The data transfer protocol of the BusNet is implemented and currently it is under testing. However, the current implementation is tailored to gather environmental data. We plan to extend the protocol to suite the collection of road surface condition data.

We are in the process of gathering acceleration data on a stretch of road with plenty of rough patches. For the initial tests we used a sensor mote placed inside a car as shown in Figure 3 and 4.

The Figure 5 shows the horizontal and vertical acceleration of the vehicle on a rough patch of road plotted against the time. The acceleration data given in the Figure 5 are raw data collected from the motes. Two baselines given in the figure indicate the horizontal and the vertical acceleration readings when the mote was placed on a level surface in the same orientation as it was mounted on the vehicle. The increasing values above a base line indicate increase of acceleration towards one direction and the decreasing values below the baseline indicate increase of acceleration towards the opposite direction. The crosses along the $y=425$ line indicate the rough patches or potholes as perceived by a passenger in the vehicle. The passenger pressed a key on the data collection terminal that we carried on the car to indicate rough patches. Because of the delays in the user response and also the delays in communicating with the mote we cannot expect the user indicated rough patches to exactly coincide with the corresponding acceleration values. We are inves- 




Figure 4: Collecting data.

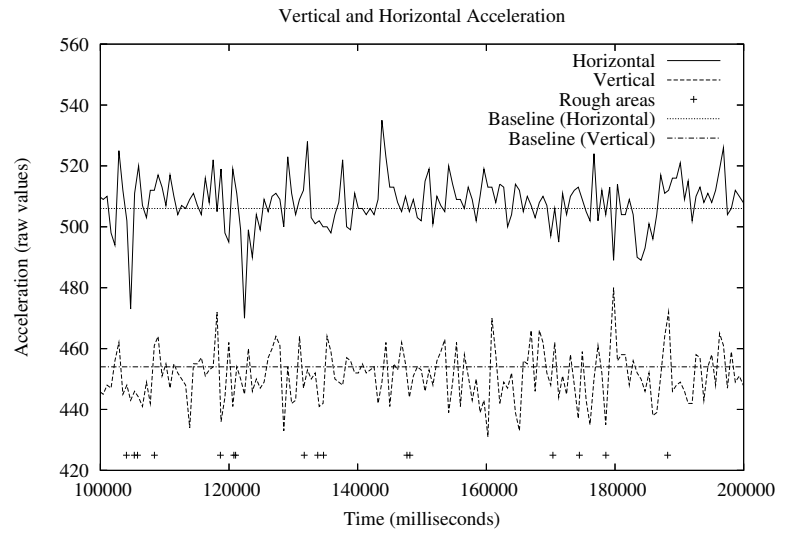

Figure 5: Horizontal and vertical acceleration for a stretch of road.

tigating better techniques to gather data on the rough patches.

We have not yet acquired GPS sensor boards that can be mounted on the motes and currently we use GPS receivers connected to PDA's for our experiments. For this particular experiment we collected GPS data from a GPS enabled PDA. The data collection terminal collected acceleration data from the mote and the GPS data from the PDA. However, for better experimental results and for the real world deployment we require GPS sensor boards.

These are preliminary results and they indicate that there is a significant change in both the vertical and horizontal components of acceleration on rough road surfaces. We are in the process of collecting more data sets and developing an analytical model.

\section{RELATED WORK}

The DakNet [6] provides digital communication services to remote villages using buses as a mechanical backhaul [7] for data transfer. The use of buses in DakNet is purely for data transfer between Internet access points and Internet kiosks in villages. In BusNet the buses provide the means to transfer data and they also collect data-buses are the moving sensors.

Data MULEs [8] architecture uses mobile entities, including buses, to collect data from sensors deployed in an environment and ferry them to access points. In contrast the buses in BusNet are not simple ferries; they also carry the sensors and the are in fact the data sources.

DakNet and the Data MULEs are examples of delay tolerant networks [4]. They are proposed as solutions for the lack of better communication infrastructure. However, the BusNet is not proposed purely as a solution for the lack of communication infrastructure. Even if a good communication infrastructure exists the data collection and transporting buses in the BusNet are the most natural form of communication infrastructure for the road surface monitoring system. It provides physical security for the sensors, reduces the cost of deploying sensors, and also the simplifies the management and maintenance of the sensors.

In ZebraNet [5] Zebras carry collars that contain sensors and the collected data are transferred to other Zebras and collection points opportunistically. This is the system that is conceptually closest to the BusNet. However, there is a crucial difference between the ZebraNet and the BusNet. While ZebraNet is an ad-hoc opportunistic network, in the BusNet there is a stable fixed infrastructure for data transfer. The bus routes are regularly serviced by scheduled buses barring a major disaster which is an exception rather than the rule. Therefore, the BusNet is a stable network and it has stable network routes. If not for the mobile buses it can even be called a fixed network.

Zhao et al. [10] also present a vehicle assisted data delivery system for vehicular ad hoc networks. In that work the vehicles are used as data carriers and the route to the destination is set up based on the ad hoc connectivity of the vehicles. In contrast, the BusNet not only delivers data it also generates data and furthermore in BusNet we use the stable transport infrastructure and does not rely on the ad hoc connectivity between vehicles.

\section{CONCLUSIONS}

This work is motivated by the observation that in Sri Lanka one of the main reasons for the deteriorated condition of the road system is the lack of continuous monitoring system of the surface condition. The BusNet, which implements a sensor network on top of the public transport network is ideal for monitoring the road surface condition; the sensor mounted buses use the very roads that we want to monitor. We designed the road surface monitoring system based on the BusNet. In this system one of the heavy users of the road system and hence a major contributor to the deterioration of the road surface helps in monitoring of the road system. A few hours of latency in BusNet is not an obstacle to this application because the data gathered on road surface condition are not needed in real time.

BusNet is a novel approach in building vehicle based data network in the sense that the sensor system which collects the data and the data themselves travel in the vehicles. Even though it was initially designed as a sensor network to monitor environmental pollution we noted its potential in road surface condition monitoring. We believe that the BusNet can be used for other sensor network applications that are delay tolerant.

The relevance of the BusNet is not limited to developing countries. It was not proposed as a solution to the lack of communication infrastructure even though it also solves that. The BusNet reduces the cost deploying a large number of sensors by replacing them with few moving sensors. It also solves the management, maintenance, and the security problems associated with a sensor network deployed over a large terrain. These are generic problems and the BusNet is of very much relevance to developed countries with good communication infrastructure. However, the road surface monitoring system that uses the BusNet is of more importance to developing countries. 


\section{ACKNOWLEDGMENT}

This project is funded by the Swedish Program for Information and Communication Technology in Developing Regions (SPIDER). We appreciate the contributions by Leif Axelsson (Volvo), Henrik Riomar (Ericsson), Martin Gustavsson (Ericsson), and I. M. K. Fernando (Department of Physics, University of Colombo). We would also like to thank the anonymous reviewers of this paper for their valuable comments.

\section{REFERENCES}

[1] A. Arora et al. Exscal: Elements of an extreme scale wireless sensor network. In RTCSA '05: Proceedings of the 11th IEEE International Conference on Embedded and Real-Time Computing Systems and Applications (RTCSA'05), pages 102-108, Washington, DC, USA, 2005. IEEE Computer Society.

[2] G. Barrenextea et al. Demo abstract: Sensorscope, an urban environmental monitoring network. In K. Langendoen and T. Voight, editors, In Parallel and Distributed Systems Report Series - Adjunct Poster/Demo Proceedings of the Fourth European Conference on Wireless and Sensor Networks, number PDS-2007-001 in Parallel and Distributed Systems Report Series. Faculty of Electrical Engineering, Mathematics and Computer Science, Delft University of Technology, 2007.

[3] Crossbow Technology Inc. MPR-MIB users manual. http: / / www . xbow . com, 2006.

[4] K. Fall. A delay-tolerant network architecture for challenged internets. In SIGCOMM '03: Proceedings of the 2003 conference on Applications, technologies, architectures, and protocols for computer communications, pages 27-34, New York, NY, USA, 2003. ACM Press.
[5] P. Juang, H. Oki, Y. Wang, M. Martonosi, L. S. Peh, and D. Rubenstein. Energy-efficient computing for wildlife tracking: design tradeoffs and early experiences with zebranet. SIGOPS Oper. Syst. Rev., 36(5):96-107, 2002.

[6] A. S. Pentland, R. Fletcher, and A. Hasson. Daknet: Rethinking connectivity in developing nations. Computer, 37(1):78-83, 2004.

[7] A. Seth, D. Kroeker, M. Zaharia, S. Guo, and S. Keshav. Low-cost communication for rural internet kiosks using mechanical backhaul. In MobiCom '06: Proceedings of the 12th annual international conference on Mobile computing and networking, pages 334-345, New York, NY, USA, 2006. ACM Press.

[8] R. Shah, S. Roy, S. Jain, and W. Brunette. Data mules: Modeling a three-tier architecture for sparse sensor networks. In In Proc. of the IEEE International Workshop on Sensor Network Protocols and Applications. IEEE, 2003.

[9] A. S. Tanenbaum, C. Gamage, and B. Crispo. Taking sensor networks from the lab to the jungle. Computer, 39(8):98-100, 2006.

[10] J. Zhao and G. Cao. VADD: Vehicle-assisted data delivery in vehicular ad hoc networks. Technical Report NAS-TR-0020-2005, Network and Security Research Center, Department of Computer Science and Engineering, Pennsylvania State University, University Park, PA, USA, July 2005.

[11] K. D. Zoysa and C. Keppitiyagama. Poster abstract: Busnet a sensor network built over a public transport system. In K. Langendoen and T. Voight, editors, Adjunct Poster/Demo Proceedings of the Fourth European Conference on Wireless and Sensor Networks, number PDS-2007-001 in Parallel and Distributed Systems Report Series. Faculty of Electrical Engineering, Mathematics and Computer Science, Delft University of Technology, 2007. 